\title{
Predictive Role of QTc Prolongation in Carbon Monoxide Poisoning-Related Delayed Neuropsychiatric Sequelae
}

\author{
Shu-Chen Liao (D, , ${ }^{1,2}$ Yan-Chiao Mao, ${ }^{2,3}$ Yao-Min Hung, \\ Ching-Hsing Lee $\mathbb{}^{1},{ }^{1}$ and Chen-Chang Yang $\mathbb{\circledR}^{2,5}$ \\ ${ }^{1}$ Department of Emergency Medicine, Chang Gung Memorial Hospital, Keelung, Taiwan \\ ${ }^{2}$ Institute of Environmental and Occupational Health Sciences, School of Medicine, National Yang-Ming University, Taipei, Taiwan \\ ${ }^{3}$ Division of Clinical Toxicology, Department of Emergency Medicine, Taichung Veterans General Hospital, Taichung, Taiwan \\ ${ }^{4}$ Department of Emergency Medicine, Kaohsiung Veterans General Hospital, Kaohsiung, Taiwan \\ ${ }^{5}$ Division of Clinical Toxicology \& Occupational Medicine, Department of Medicine, Taipei Veterans General Hospital, Taipei, Taiwan
}

Correspondence should be addressed to Chen-Chang Yang; ccyang@vghtpe.gov.tw

Received 21 May 2018; Revised 17 August 2018; Accepted 29 August 2018; Published 25 September 2018

Academic Editor: Gelin Xu

Copyright (C) 2018 Shu-Chen Liao et al. This is an open access article distributed under the Creative Commons Attribution License, which permits unrestricted use, distribution, and reproduction in any medium, provided the original work is properly cited.

\begin{abstract}
Objective. Delayed neuropsychiatric sequelae (DNS) are serious complications of carbon monoxide (CO) poisoning that adversely affect poisoned patients' quality of life as well as socioeconomic status. This study aimed to determine clinical predictors of DNS in patients with CO poisoning. Methods. This retrospective study included all CO-poisoned patients admitted to the emergency department (ED) of Linkou Chang Gung Memorial Hospital in Taiwan from 1 January 2009 to 31 December 2015. The medical records of all patients with CO poisoning were carefully reviewed, and relevant data were abstracted into a standardised form. Univariate and multivariate logistic regression models were used to identify predictors of DNS after CO poisoning. Receiver operating characteristic (ROC) curve analysis was used to determine the ideal cut-off value for continuous variables that predict the development of DNS. Results. A total of 760 patients with CO poisoning were identified during the study period. Among them, 466 were eligible for the analysis of predictors of DNS. In multivariate analysis, Glasgow Coma Scale $<9$ (odds ratio [OR], 2.74; 95\% confidence interval $[\mathrm{CI}], 1.21-6.21)$, transient loss of consciousness (OR, 3.59; 95\% CI, 1.31-9.79), longer duration from CO exposure to ED presentation (OR, 1.05; 95\% CI, 1.03-1.08), and corrected QT (QTc) prolongation (OR, 2.61; 95\% CI, 1.21-5.61) were found to be associated with a higher risk of DNS. The area under the ROC curve (AUC) for QTc interval measured within $6 \mathrm{~h}$ after exposure best predicted the development of DNS, with a result of 0.729 (95\% CI 0.660-0.791). Moreover, the best cut-off value of the QTc interval was $471 \mathrm{~ms}$, with a sensitivity of $53.3 \%$ and a specificity of $85.1 \%$. Conclusions. We identified several potential predictors of DNS following CO poisoning. Among them, QTc prolongation found within $6 \mathrm{~h}$ after exposure is a novel predictor of DNS, which may be helpful in the future care of patients with $\mathrm{CO}$ poisoning.
\end{abstract}

\section{Introduction}

Carbon monoxide (CO) is a colourless, odourless, and highly toxic gas that is produced during incomplete carbon combustion. CO poisoning can occur following the inhalation of partially combusted charcoal briquettes, fuel gas, or oil in a poorly ventilated place. Domestic exposure can occur through incomplete combustion in gas-furnace water heaters, through inhalation of exhaust gas from automobiles, or in fire accidents $[1,2]$.

Since 2002, CO poisoning has been one of the leading causes of death by suicide in Taiwan. According to the
National Suicide Database of Taiwan, the number of deaths by suicide using 'gases in domestic use' or 'other gases and vapours' has risen year-by-year, peaking in 2006. The number of deaths from burning charcoal has declined since 2012 after the government enacted regulation on the sale of charcoal (http://dep.mohw.gov.tw/DOMHAOH/cp-332-8883-107 .html).

Most patients with $\mathrm{CO}$ poisoning present to the emergency department (ED) with nonspecific symptoms such as headache, dizziness, malaise, asthenia, nausea, vomiting, flu-like syndrome, transient loss of consciousness, altered mental status, chest tightness, palpitation, and dyspnoea. 
TABLE 1: Previously reported signs and symptoms of DNS [3].

\begin{tabular}{lc}
\hline Neurological sequelae & Cognitive and psychological sequelae \\
\hline Parkinson-like syndromes & Concentration deficit \\
Gait and motor disturbances & Memory loss \\
Bradykinesia & Cognitive impairment \\
Intention tremor & Dementia \\
Myoclonus & Personality changes \\
Dyspraxia & Anxiety \\
Dysphasia & Extreme emotional lability \\
Ataxia & Psychosis \\
Postural instability & Depression \\
Vertigo & Mania \\
Cortical blindness & Insomnia \\
Hearing loss, tinnitus & \\
Chorea & \\
EEG abnormalities & \\
Epilepsy & \\
Peripheral neuropathies & \\
Recurrent headache & \\
Fecal/urinary incontinence & \\
\hline
\end{tabular}

Life-threatening cardiac arrhythmia, seizures, and coma may also develop in cases of severe $\mathrm{CO}$ poisoning. In the absence of a clear medical history, the aforementioned manifestations may create diagnostic challenges for emergency physicians $[3,4]$.

In addition to causing acute morbidity and mortality, CO poisoning can cause delayed neuropsychiatric sequelae (DNS) that occur days or weeks after initial complete clinical recovery from acute poisoning [5]. The mechanisms underlying DNS remain unclear; however, progressive inflammation may be involved. CO diffuses quickly into the blood via the lungs and causes direct tissue hypoxaemia through the formation of carboxyhaemoglobin $(\mathrm{COHb})$ and a leftward shift of the oxyhaemoglobin dissociation curve. CO also binds to haeme-containing proteins such as cytochrome $c$ oxidase and platelet haemoprotein. All the aforementioned mechanisms can induce a chain reaction of inflammation that increases the production of reactive oxygen species and induces oxidative stress, lipid peroxidation, and apoptosis [57].

The incidence of DNS following CO poisoning varies widely from $3 \%$ to $46 \%$, and the lag time ranges between 2 days and 6 weeks after exposure [8-11]. Because of the lack of standard diagnostic criteria, most reported DNS include a broad spectrum of neurological deficits, cognitive impairments, and psychological disorders (Table 1) [3]. In patients with CO poisoning, the occurrence of DNS can result in decreased ability to conduct instrumental as well as other activities of daily living [12]. Thus, the prompt identification of CO-poisoned patients who are at risk of DNS is vital to ensure that effective treatment is provided to reduce the risk of DNS.

Studies have revealed various clinical predictors of DNS following $\mathrm{CO}$ poisoning, including age $>36$ years, long exposure to CO, higher COHb levels (>25\%), damage in the globus pallidus or white matter at initial ED presentation, Glasgow Coma Scale (GCS) score $<9$, seizures, systolic blood pressure $<90 \mathrm{mmHg}$ at ED presentation, elevated creatine phosphokinase concentration, leukocytosis, and a positive Babinski reflex [1, 3, 4, 8-14]. However, these findings remain inconclusive.

In acute $\mathrm{CO}$ poisoning, numerous electrocardiogram (ECG) alterations, such as P-wave dispersion, corrected QT (QTc) dispersion, and QTc prolongation, have been reported [2,15-18], which might be related to life-threatening arrhythmias. The possible mechanism of ECG alterations in CO poisoning is related to S-nitrosylation of the $\mathrm{Na}^{+}$channel, which increases the late inward $\mathrm{Na}^{+}$current, resulting in prolongation of the action potential and the associated intracellular $\mathrm{Ca}^{2+}$ transients, subsequently causing disruption of repolarisation and prolongation of the QT interval. However, no study has determined the relation between acute ECG alterations and DNS [19]. To elucidate the predictive role of ECG alterations, particularly QTc prolongation, and to identify other clinical predictors of CO-related DNS, we conducted a retrospective cohort study of acute $\mathrm{CO}$-poisoned patients in a tertiary medical center in Taiwan.

\section{Methods}

2.1. Ethics. The study protocol was approved by the Institutional Review Board of Chang Gung Medical Foundation (104-7628C) and was granted permission by the Medical Ethics Committee of Chang Gung Memorial Hospital.

2.2. Study Design, Study Population, and Participant Selection. A retrospective medical record review was conducted for all CO-poisoned patients (ICD-9-CM code as 986) admitted to the ED of Linkou Chang Gung Memorial Hospital from 1 January 2009 to 31 December 2015. COHb levels were measured using an arterial blood gas analyser with a CO oximeter. Patients enrolled into this study were required to meet at least one of the following criteria: (1) a COHb level of $>5 \%$ in nonsmokers and $>10 \%$ in smokers at presentation and (2) delayed presentation with an unambiguous history of CO poisoning, such as being found in a confined space with burning charcoal, exposure to car or machine exhaust, or living in a place with a faulty water heater. The medical records of all patients were reviewed, and information on clinical symptoms and laboratory findings on admission to the ED, circumstances of exposure, treatment received, clinical outcomes, and other information available at the ED were abstracted.

2.3. Data Collection and Definition of Variables. All patients were provided with $100 \%$ oxygen from a "nonrebreathing" facemask (NRM) as soon as CO poisoning was suspected at the ED or prehospital setting when attended by emergency services. Information on the following variables was collected on admission to the ED: age; sex; psychiatric history; referral institution; intentional or accidental exposure; CO exposure source; vital signs; Taiwan Triage and Acuity Scale (TTAS); concomitant use of tranquilisers; transient 
loss of consciousness; duration of loss of consciousness; duration from $\mathrm{CO}$ exposure to $\mathrm{ED}$ admission; evidence of myocardial injury (defined as electrocardiographic signs of ischaemia/arrhythmias and cardiac enzyme elevation); ECG; images such as brain computed tomography (CT); treatment modality (hyperbaric oxygen therapy(HBOT) at 2.5 atmosphere absolute for 90 minutes/session or 100\% normobaric oxygen therapy through NRM); number of sessions of HBOT; duration from $\mathrm{CO}$ exposure to HBOT administration; and length of hospital and intensive care unit stay. The results of blood tests on ED visit and admission, including complete blood cell count, arterial blood gas analysis, COHb level, troponin I, creatine phosphokinase, and serum alcohol level, were also collected. Data on urine benzodiazepine and illicit drug screening were abstracted as well.

ECG findings were further analysed to calculate the QTc interval. The QTc interval is the measured QT interval corrected for heart rate using Bazett's formula (QT/RRK). QTc prolongation has been defined as follows: longer than $440 \mathrm{~ms}$ in men and longer than $460 \mathrm{~ms}$ in women $[2,20]$.

All CO-poisoned patients received 100\% oxygen supplement through NRM upon ED arrival. Some patients received HBOT after consultation with an HBOT specialist following the management guideline of $\mathrm{CO}$ poisoning of the American Undersea and Hyperbaric Medical Society, 13th Edition (https://www.uhms.org/carbon-monoxide-poisoning/carbon-monoxide-poisoning.html).

DNS were defined as the recurrence of original symptoms or the development of new symptoms such as difficulty concentrating, lethargy, emotional lability, mutism, amnestic syndromes, dementia, psychomotor retardation, Parkinsonism, apraxia, unsteady gait, and urinary incontinence, within 2-42 days after CO poisoning [3, 5, 6, 10-12, 14]. All poisoned patients were invited to the follow-up visits from hospital discharge in outpatient clinic. A comprehensive neurological exam was performed and also the mental status examination during the visit. The follow-up duration to observe the development of DNS was at least 6 months.

2.4. Statistical Analysis. Demographic and clinical data of the patients with and without DNS were compared using Fisher's exact test for categorical variables or the Mann-Whitney $U$ test for continuous variables because most continuous variables were not normally distributed. To investigate the factors associated with DNS development, we introduced the variables with $P<0.2$ in univariate analyses into a multivariable logistic regression model with stepwise selection. The ability of QTc to predict DNS development was evaluated using receiver operating characteristic (ROC) curve analysis. Finally, the proportion of QTc prolongation in patients with and without DNS stratified by duration from CO exposure to ECG recording was compared using Fisher's exact test. Data analysis was conducted using MedCalc Statistical Software version 13.1.2 (MedCalc Software bvba, Ostend, Belgium; http://www.medcalc.org; 2014). All odds ratios (ORs) were reported with relevant $95 \%$ confidence intervals (CIs). $P<$ 0.05 was considered statistically significant.

\section{Results}

A total of 760 patients with $\mathrm{CO}$ poisoning who met the inclusion criteria of this study were admitted to the ED of Linkou Chang Gung Memorial Hospital from 2009 to 2015. Among them, 466 patients were eligible for further DNS detection (Supplementary Table 1). A total of 294 patients were excluded because we were unable to confirm or exclude the diagnosis of DNS. Among the excluded patients, 19 were in a persistent vegetative state or unresponsive wakefulness; 12 died during ED admission; 3 were discharged from ED in a moribund status upon family's request; and contact was lost with 260 after discharge from the ED.

Among the 466 included patients, almost half were men $(230 / 466,49.4 \%)$, and the median age was 33 years (interquartile: $22-45$ years). A total of 223 patients (47.9\%) were transferred to our ED from another medical institution; 190 patients (40.8\%) had been attempting suicide. Inappropriately ventilated gas from heating appliances was the most common cause of CO poisoning $(238 / 466,51.1 \%)$, followed by charcoal burning (196/466, 42.1\%). Most patients presented with clear consciousness upon arrival, and $62(62 / 466,13.3 \%)$ had poor consciousness with a GCS of $<9$. More than half had experienced transient loss of consciousness after $\mathrm{CO}$ poisoning $(292 / 466,62.7 \%)$, and most patients presented with loss of consciousness for $<6 \mathrm{~h}(210 / 292,71.9 \%)$. The median initial COHb level upon admission was 10.1\% (median: $10.1 \%$, interquartile: $4.0 \%-22.6 \%$ ), and slightly over half of patients had leukocytosis $(245 / 444,56.5 \%)$. Laboratory test results revealed that $64(18 \%)$ patients had myocardial injury. The median time from $\mathrm{CO}$ exposure to $\mathrm{ED}$ admission and to ECG recording was 4.0 and $5.1 \mathrm{~h}$, respectively. A 12-lead ECG revealed that $42.1 \%$ of patients had QTc prolongation. A total of 279 (59.9\%) received HBOT, with a median total of 3 sessions (Table 2).

3.1. Univariate Analysis of Potential Risk Factors for DNS. Among the 466 patients with adequate follow-up period to detect DNS, DNS diagnosis was confirmed in 62 (13.3\%), with a median duration from $\mathrm{CO}$ exposure of 10 days (interquartile: 7-24 days). Univariate analysis identified the following risk factors for DNS: old age $(P=0.005)$; psychiatric history $(P=0.047)$; poor consciousness level at ED presentation and GCS $<9$ at admission $(P<0.001)$; higher TTAS $(P=0.014)$; intentional poisoning $(P<0.001)$; charcoal burning $(P<$ $0.001)$; concomitant tranquiliser use $(P=0.015)$; transient loss of consciousness and longer duration of loss of consciousness $(P<0.001)$; longer duration from $\mathrm{CO}$ exposure to $\mathrm{ED}$ admission; and ECG revealing QTc prolongation. Laboratory test results revealed that the presence of myocardial injury (Trop-I $>0.5 \mathrm{ng} / \mathrm{mL}$ ) and leukocytosis $(\mathrm{WBC}>10.000 / \mathrm{mL})$ was also associated with DNS $(P<0.001$ and $P=0.004$, respectively) (Table 2).

3.2. Multivariable Logistic Regression of Risk Factors for DNS. Variables with $P<0.2$ in univariate analyses (as shown in Table 2) were introduced into the multivariable model using stepwise selection. The results demonstrated that GCS <9 (OR, 2.74; 95\% CI, 1.21-6.21), transient loss of consciousness 
TABLE 2: Demographic and clinical data of patients with and without DNS.

\begin{tabular}{|c|c|c|c|c|}
\hline Variables & $\begin{array}{c}\text { Total } \\
(N=466)\end{array}$ & $\begin{array}{c}\text { DNS } \\
(n=62)\end{array}$ & $\begin{array}{r}\text { Non-DNS } \\
(n=404) \\
\end{array}$ & $P$ value \\
\hline Male gender & $230(49.4)$ & $35(56.5)$ & $195(48.3)$ & 0.275 \\
\hline Age (years) & $33[22,45]$ & $41[26,52]$ & $32[21,43]$ & 0.005 \\
\hline Psychiatric history & $82(17.6)$ & $17(27.4)$ & $65(16.1)$ & 0.047 \\
\hline Pulse, beats/min & $100[84,114]$ & $96[84,110]$ & $100[85,115]$ & 0.346 \\
\hline Pulse $>100$ beats/min & $216(47.7)$ & $25(41.0)$ & $191(48.7)$ & 0.273 \\
\hline Glasgow Coma Score (GCS) & $15[14,15]$ & $14[7,15]$ & $15[15,15]$ & $<0.001$ \\
\hline GCS less than 9 & $62(13.3)$ & $19(30.6)$ & $43(10.6)$ & $<0.001$ \\
\hline Triage scale & & & & 0.014 \\
\hline 1, resuscitation & $74(15.9)$ & $18(29.0)$ & $56(13.9)$ & \\
\hline 2 , emergent & $290(62.2)$ & $33(53.2)$ & $257(63.6)$ & \\
\hline 3 , urgent & $94(20.2)$ & $9(14.5)$ & $85(21.0)$ & \\
\hline 4/5 less urgent/not urgent & $8(1.7)$ & $2(3.2)$ & $6(1.5)$ & \\
\hline Transferred from outside institution & $223(47.9)$ & $37(59.7)$ & $186(46.0)$ & 0.056 \\
\hline Attempted suicide & $190(40.8)$ & $42(67.7)$ & $148(36.6)$ & $<0.001$ \\
\hline Source of CO poisoning & & & & $<0.001$ \\
\hline Charcoal burning & $196(42.1)$ & $45(72.6)$ & $151(37.4)$ & \\
\hline Inappropriately ventilated gas heating appliances & $238(51.1)$ & $13(21.0)$ & $225(55.7)$ & \\
\hline Others & $32(6.9)$ & $4(6.5)$ & $28(6.9)$ & \\
\hline Concomitant use with tranquilizer & $63(13.5)$ & $15(24.2)$ & $48(11.9)$ & 0.015 \\
\hline Transient loss of consciousness & $292(62.7)$ & $55(88.7)$ & $237(58.7)$ & $<0.001$ \\
\hline Duration of loss of consciousness & & & & $<0.001$ \\
\hline$<6$ hours & $210(71.9)$ & $25(45.5)$ & $185(78.1)$ & \\
\hline 6-12 hours & $28(9.6)$ & $5(9.1)$ & $23(9.7)$ & \\
\hline 13-24 hours & $20(6.8)$ & $13(23.6)$ & $7(3.0)$ & \\
\hline$>24$ hours & $16(5.5)$ & $10(18.2)$ & $6(2.5)$ & \\
\hline$>48$ hours & $18(6.2)$ & $2(3.6)$ & $16(6.8)$ & \\
\hline Leukocytosis & $245(56.5)$ & $43(74.1)$ & $202(53.7)$ & 0.004 \\
\hline Metabolic acidosis & $67(19.1)$ & $9(19.6)$ & $58(19.1)$ & 1.000 \\
\hline $\mathrm{COHb}, \%$ & $10.1[4.0,22.6]$ & $7.4[3.1,19.8]$ & $10.9[4.2,22.7]$ & 0.095 \\
\hline Troponin I, ng/mL & $0.20[0.01,1.83]$ & $0.93[0.15,4.30]$ & $0.14[0.01,1.72]$ & 0.001 \\
\hline Evidence of myocardial injury & $64(18.0)$ & $21(42.9)$ & $43(14.0)$ & $<0.001$ \\
\hline Time from CO exposure to ED (hours) & $4.0[2.5,8.0]$ & $9.8[4.8,21.8]$ & $4.0[2.5,7.0]$ & $<0.001$ \\
\hline Time from CO exposure to ECG recording (hours) & $5.1[3.1,8.5]$ & $8.3[4.5,21.1]$ & $4.8[3.0,7.5]$ & $<0.001$ \\
\hline QTc prolongation & $149(42.1)$ & $37(69.8)$ & $112(37.2)$ & $<0.001$ \\
\hline Brain CT imaging study at first medical institution & $131(28.1)$ & $40(64.5)$ & $91(22.5)$ & $<0.001$ \\
\hline HBOT & $279(59.9)$ & $47(75.8)$ & $232(57.4)$ & 0.008 \\
\hline Number of HBOT sessions & $3[1,3]$ & $4[2,6]$ & $2[1,3]$ & $<0.001$ \\
\hline Length of hospital stay (days) & $1[0,3]$ & $5[1,11]$ & $1[0,3]$ & $<0.001$ \\
\hline ICU stay (days) & $26(5.6)$ & $10(16.1)$ & $16(4.0)$ & 0.001 \\
\hline $\begin{array}{l}\text { Lucid interval, duration from exposure to DNS } \\
\text { (days) }\end{array}$ & NA & $10[7,24]$ & NA & NA \\
\hline
\end{tabular}

Continuous data were expressed as median $\left(25^{\text {th }}\right.$ and $75^{\text {th }}$ percentiles); categorical data were presented as frequency (proportion).

(OR, 3.59; 95\% CI, 1.31-9.79), longer duration from exposure to $\mathrm{ED}$ admission (OR, 1.05; 95\% CI, 1.03-1.08), and QTc prolongation (OR, 2.61; 95\% CI, 1.21-5.61) were associated with a higher risk of DNS. In addition, there was a trend indicating that increased age might be correlated with DNS development (OR, 1.18; $P=0.089)$ (Table 3).

Table 4 displays summaries of the ROC curves for the discrimination ability of QTc for DNS development stratified 
TABLE 3: Factors associated with DNS development in multivariate logistic regression analysis.

\begin{tabular}{lcc}
\hline Variables & Odds ratio (95\% CI) & $P$ value \\
\hline Age (per 10 years) & $1.18(0.98-1.42)$ & 0.089 \\
GCS $<9$ & $2.74(1.21-6.21)$ & 0.016 \\
Transient loss of consciousness & $3.59(1.31-9.79)$ & 0.013 \\
Duration from CO exposure to ED (hours) & $1.05(1.03-1.08)$ & $<0.001$ \\
QTc prolongation & & 0.014 \\
$\quad$ Yes vs. No & $2.61(1.21-5.61)$ & 0.653 \\
$\quad$ Yes vs. Unknown & $0.77(0.24-2.45)$ & \\
\hline
\end{tabular}

CI, confidence interval.

TABLE 4: ROC curves of QTc for discriminating DNS stratified by duration from CO exposure to ECG recording.

\begin{tabular}{llcc}
\hline Hour from exposure to ECG recording & AUC & 95\% Confidence Interval of AUC & $P$ value \\
\hline$<2$ hrs. & 0.747 & 0.563 to 0.883 & 0.036 \\
$2-3$ hrs. & 0.740 & 0.632 to 0.831 & 0.034 \\
$4-5$ hrs. & 0.743 & 0.630 to 0.837 & 0.007 \\
$6-7$ hrs. & 0.683 & 0.531 to 0.811 & 0.221 \\
$\geq 8$ hrs. & 0.518 & 0.408 to 0.626 & 0.821 \\
\hline
\end{tabular}

AUC, area under the curve.

by duration from CO exposure to ECG recording. The result revealed that the discrimination ability was significant and acceptable for durations $<2 \mathrm{~h}(\mathrm{AUC}=0.747), 2-3 \mathrm{~h}(\mathrm{AUC}=$ $0.740)$, and $4-5 \mathrm{~h}(\mathrm{AUC}=0.743)$. The discrimination ability was poorer for durations of $6-7 \mathrm{~h}$ and $\geq 8 \mathrm{~h}$. Figure 1 plots the ROC curve of QTc for discriminating DNS within $6 \mathrm{~h}$ from exposure to ECG recording. The relevant AUC was 0.729 (95\% CI, 0.660-0.791), and the best cut-off point was $471 \mathrm{~ms}$, with a sensitivity of $53.3 \%$ and a specificity of $85.1 \%$, a positive predictive value of $23.5 \%$, and a negative predictive value of $95.5 \%$. Similarly, we found that the proportion of QTc prolongation in patients with and without DNS was statistically significant in periods $<2 \mathrm{~h}(100 \%$ versus $31 \%, P$ $=0.044), 2-3 \mathrm{~h}(100 \%$ versus $44.9 \%, P=0.047)$, and $4-5 \mathrm{~h}$ (87.5\% versus $26.9 \%, P=0.001$ ) from CO exposure to ECG recording (Figure 2).

\section{Discussion}

Neurological deficits and cognitive disorders impede the ability of patients to exercise self-care as well to participate in occupational and social activities; therefore, such conditions can affect the lives of not only patients but also their families [4]. Several predictors of DNS following CO poisoning have been proposed: GCS <9 [3], MMSE scores, positive findings on brain CT images [10], transient loss of consciousness, age $>36$ years, $\mathrm{COHb}$ levels $\geq 25 \%$ [5], serious conscious disturbance at ED presentation, elevated creatine kinase levels, elevated creatine kinase-MB and lactate dehydrogenase levels, and low Global Assessment Scale scores [1].

This study aimed to identify predictors of DNS after CO poisoning, which may be of use to emergency physicians. Furthermore, to the best of our knowledge, this retrospective study enrolled the largest number of patients to date for any such study.

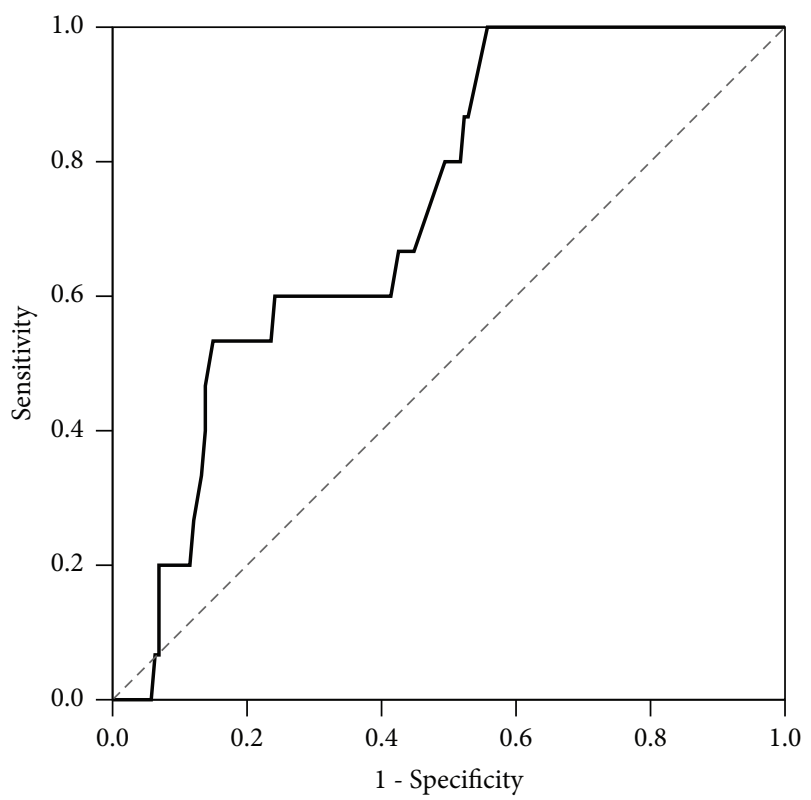

FIGURE 1: ROC curve of QTc for discriminating DNS by ECG recording performed within 6 hours after $\mathrm{CO}$ exposure. The area under ROC (AUC) was 0.729 (95\% CI: 0.660-0.791). The best cutoff point was $471 \mathrm{~ms}$ with a sensitivity of $53.3 \%$, a specificity of $85.1 \%$, a positive predictive value of $23.5 \%$, and a negative predictive value of $95.5 \%$.

The severity of $\mathrm{CO}$ poisoning is determined by the duration of $\mathrm{CO}$ exposure and the concentration of $\mathrm{CO}$ in the atmosphere, but not by the $\mathrm{COHb}$ level at admission $[1,3,21-23]$. COHb has a fundamental diagnostic role, but the lack of its prognostic value has been confirmed by many studies. COHb levels are influenced by several factors, such as 


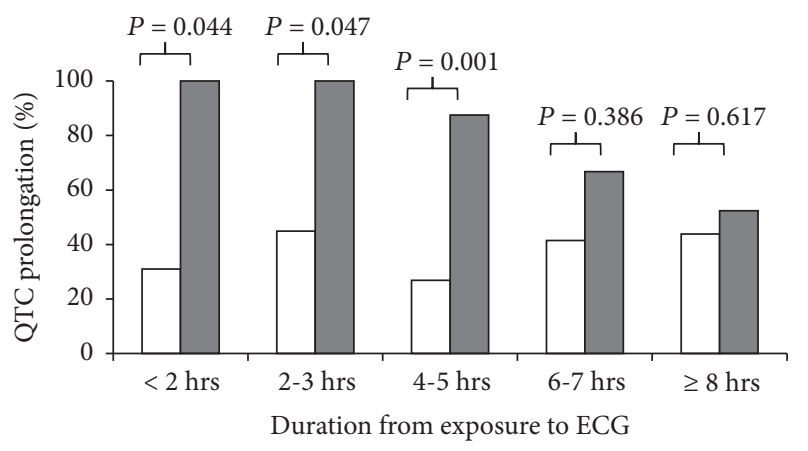

$\square$ Non-DNS

$\square$ DNS

FIGURE 2: QTc prolongation in patients with and without DNS stratified by duration from CO exposure to ECG recording.

prehospital oxygen administration and time elapsed between exposure cessation and hospital admission [3].

In this study, we determined that GCS $<9$, transient loss of consciousness, longer duration from $\mathrm{CO}$ exposure to ED admission, and ECG revealing QTc prolongation were independent predictors of DNS in CO-poisoned patients (Table 3). Among these factors, other studies have reported lower GCS and transient loss of consciousness as predictors of DNS in CO-poisoned patients $[3,8,10]$, but this study provides the first indication of longer duration from $\mathrm{CO}$ exposure to ED admission and ECG revealing QTc prolongation as predictors. This finding may help emergency physicians make prompt diagnoses of DNS and arrange further treatment for patients with $\mathrm{CO}$ poisoning. For patients at a high risk of DNS, intensive treatment and monitoring, including earlier HBOT and follow-up for neuropsychiatric sequelae, should be considered.

A longer duration from $\mathrm{CO}$ exposure to $\mathrm{ED}$ admission indicates prolonged $\mathrm{CO}$ exposure or a longer interval from exposure cessation to hospital treatment. Longer exposure to $\mathrm{CO}$ is one of the determinants of severity, and a longer interval from $\mathrm{CO}$ exposure to ED admission implies a delay in treatment with any type of oxygen supplement such as normobaric oxygen or HBOT.

Acute $\mathrm{CO}$ poisoning frequently affects the repolarisation of myocardium electrophysiology, resulting in abnormal ECG findings such as QTc prolongation and QTc dispersion $[16,18,19,24]$. These changes may lead to life-threatening cardiac arrhythmia or proarrhythmia effects [19]. Ventricular repolarisation can be evaluated by measuring the QT interval, QTc interval, and QTc dispersion. In in vitro studies, CO poisoning resulted in elevated $\mathrm{NO}$ levels in myocytes, causing S-nitrosylation of the $\mathrm{Na}^{+}$channel and increasing the late component of the inward $\mathrm{Na}^{+}$current. This subsequently resulted in prolongation of the action potential and the associated intracellular $\mathrm{Ca}_{2}{ }^{+}$transients, leading to disruption of ventricular myocyte repolarisation and QT prolongation $[2,15]$. Studies have also suggested that prolonged QTc and QTc dispersion increased the risk of atrial and ventricular arrhythmia in patients with CO poisoning $[2,16]$. However, no study has linked QTc interval or QTc dispersion with the prediction of DNS in patients with CO poisoning. Theoretically, the more severe a case of $\mathrm{CO}$ poisoning is, the higher the chance of DNS development. However, to the best of our knowledge, few biomarkers or poisoning severity scores are available that can be used as a pragmatic surrogate for $\mathrm{CO}$ poisoning severity in the ED setting. Based on the findings of the present study, QTc prolongation may be more representative of $\mathrm{CO}$ poisoning severity than previously thought.

QTc interval and its dispersion were dynamic parameters for CO poisoning in the present study. QTc prolongation was found at admission and resolved significantly between 6 and $24 \mathrm{~h}$ after admission $[2,17]$. Our data also indicated that conducting ECG within $6 \mathrm{~h}$ of CO exposure had a discernible effect on the prediction of DNS, with an AUC of $0.729(95 \%$ CI: 0.660-0.791) and a best cut-off point of $471 \mathrm{~ms}$ of the QTc interval (Figure 1). However, no statistical significance was revealed for ECG conducted $>6 \mathrm{~h}$ after $\mathrm{CO}$ exposure (Figure 2). This is reasonable because $\mathrm{CO}$ is eliminated through pulmonary circulation, and the elimination half-life of $\mathrm{CO}$ is approximately 300,90 , and 30 min under the breathing of room air, $100 \%$ oxygen via a reservoir or NRM facemask, and HBOT, respectively [9]. In our study, the median time from $\mathrm{CO}$ exposure to $\mathrm{ED}$ admission was $4 \mathrm{~h}$ (interquartile: 2.5-8 h). Nevertheless, all CO-poisoned patients had received prehospital normobaric oxygen therapy, which could lead to pulmonary decontamination of $\mathrm{CO}$ and subsequently confounded the results of ECG.

This study has several limitations. First of all, there has been a steady decline in the number of follow-up visits after discharging a patient from the ED, which has resulted in a decreased ability to detect DNS and might have adversely affected the statistical power of this study. Secondly, we adopted a symptom-based diagnostic criteria of DNS because of the lack of standard ones. Previous studies employed bundle battery of test [5], Folstein Mini-Mental Status Examination (MMSE) [10], Hamilton Depression Rating Scale (HDRS), Beck Anxiety Inventory, Wechsler Memory ScaleRevised (WMS-R), and Verbal Memory Process Test (VMPT) [4] as the diagnostic criteria of DNS, but these tests were still inconclusive. Thirdly, if we could obtain serial ECG followups in every single patient, we wound be able to detect dynamic QTc duration and provide more strong evidence on the usefulness of QTc prolongation in predicting DNS following $\mathrm{CO}$ poisoning. Finally, since this was a retrospective study and the data were abstracted from a chart review, the clinical presentations or medical records might not have been completely documented, which could then lead to the loss of statistical significance in certain potential predictors of DNS.

\section{Conclusion}

DNS is the most serious complication after patient recovery from acute $\mathrm{CO}$ poisoning. DNS represents a challenge to emergency physicians because no established criteria exist for determining the risk of DNS in the ED setting. We conducted a retrospective study to identify independent predictors of DNS after CO poisoning. The results revealed that GCS 
$<9$, transient loss of consciousness, longer duration from CO exposure to ED presentation, and ECG revealing QTc prolongation are independent predictors for DNS of $\mathrm{CO}$ poisoning. Of these factors, ECG revealing QTc prolongation within 6 hours after $\mathrm{CO}$ exposure is first identified as a predictor of DNS. Early detection of CO-poisoned patients with a high DNS risk at the ED may help physicians plan better therapeutic strategies and arrange suitable follow-up treatment for the patients.

\section{Data Availability}

The data used to support the findings of this study are restricted by the Chang Gung Medical Foundation Institutional Review Board in order to protect patient privacy. Data are available from the corresponding author: ChenChang Yang for researchers who meet the criteria for access to confidential data.

\section{Disclosure}

The funder had no role in the study design, data collection and analysis, decision to publish, or preparation of the manuscript.

\section{Conflicts of Interest}

The authors declare that they have no conflicts of interest and have nothing to disclose about financial or nonfinancial conflicts of interest related to the content discussed in this manuscript.

\section{Authors' Contributions}

Shu-Chen Liao, Chen-Chang Yang, and Ching-Hsing Lee conceived the study and obtained research funding. ShuChen Liao and Chen-Chang Yang supervised the conduct of this study and data collection. Yan-Chiao Mao, Shu-Chen Liao, and Yao-Min Hung provided statistical advice on study design and analysed the data. Shu-Chen Liao drafted the manuscript, and all authors contributed substantially to its revision. Chen-Chang Yang takes responsibility for the paper as a whole.

\section{Acknowledgments}

This study was funded by Chang Gung Memorial Hospital Research Grant (CMRPG2E0091). The authors are grateful to the physicians and nursing staff of HBOT Center of Linko Chang Gung Memorial Hospital for their valuable contribution.

\section{Supplementary Materials}

Supplement Table 1. Demographic and clinical data of patients with and without DNS detection. Supplement Table 1 lists the demographic and clinical data of patients with and without DNS detection. Compared to patients without DNS detection, those with DNS detection had the following characteristics: higher proportion of pulse $>100$ beat $/ \mathrm{min}$, higher proportion of GCS less than 9, lower score of Triage scale, less likely to be transferred from outside institution, higher proportion of evidence of myocardial injury, higher proportion of brain CT at first medical institution, less likely to receive $\mathrm{HBO} 2$ therapy, receiving a greater number of $\mathrm{HBO} 2$ sessions, having longer length of hospital stay, and more likely to stay in ICU. Supplement Table 2. Demographic and clinical data of patients with and without electrocardiography (ECG). Supplement Table 2 lists the demographic and clinical data of patients with and without ECG. Compared to patients without ECG, those with ECG detection had the following characteristics: less likely to be male, being older, lower proportion of pulse $>100$ beat/min, higher proportion of leukocytosis, higher proportion of evidence of myocardial injury, and more likely to have QTc prolongation. (Supplementary Materials)

\section{References}

[1] K. Kudo, K. Otsuka, J. Yagi et al., "Predictors for delayed encephalopathy following acute carbon monoxide poisoning," BMC Emergency Medicine, vol. 14, no. 1, 2014.

[2] B. Yelken, "The assessment of QT intervals in acute carbon monoxide poisoning," Anadolu Kardiyoloji Dergisi, vol. 9, no. 5, pp. 397-400, 2009.

[3] G. Pepe, M. Castelli, P. Nazerian et al., "Delayed neuropsychological sequelae after carbon monoxide poisoning: predictive risk factors in the Emergency Department. A retrospective study," Scandinavian Journal of Trauma, Resuscitation and Emergency Medicine, vol. 19, article no. 16, 2011.

[4] Y. Katirci, H. Kandiş, Ş. Aslan, and İ. Kirpinar, "Neuropsychiatric disorders and risk factors in carbon monoxide intoxication," Toxicology \& Industrial Health, vol. 27, no. 5, pp. 397-406, 2011.

[5] L. K. Weaver, R. O. Hopkins, and k. J. Chan, "Hyperbaric oxygen for acute carbon monoxide poisoning," The New England Journal of Medicine, vol. 347, no. 14, pp. 1057-1067, 2002.

[6] L. K. Weaver, "Carbon monoxide poisoning," The New England Journal of Medicine, vol. 360, no. 12, pp. 1217-1225, 2009.

[7] J. A. Guzman, “Carbon Monoxide Poisoning," Critical Care Clinics, vol. 28, no. 4, pp. 537-548, 2012.

[8] L. K. Weaver, K. J. Valentine, and R. O. Hopkins, "Carbon monoxide poisoning: risk factors for cognitive sequelae and the role of hyperbaric oxygen," American Journal of Respiratory and Critical Care Medicine, vol. 176, no. 5, pp. 491-497, 2007.

[9] Y. C. Chang, H. Y. Lee, J. L. Huang, C. H. Chiu, C. L. Chen, and C. T. Wu, "Risk factors and outcome analysis in children with carbon monoxide poisoning," Pediatrics and Neonatology, 2016.

[10] H.-L. Ku, K.-C. Yang, Y.-C. Lee, M.-B. Lee, and Y.-H. Chou, "Predictors of carbon monoxide poisoning-induced delayed neuropsychological sequelae," General Hospital Psychiatry, vol. 32, no. 3, pp. 310-314, 2010.

[11] Jian-Fang Zou, Qiming Guo, Hua Shao et al., "A Positive Babinski Reflex Predicts Delayed Neuropsychiatric Sequelae in Chinese Patients with Carbon Monoxide Poisoning," BioMed Research International, vol. 2014, Article ID 814736, 5 pages, 2014.

[12] H. Hu, X. Pan, Y. Wan, Q. Zhang, and W. Liang, "Factors affecting the prognosis of patients with delayed encephalopathy 
after acute carbon monoxide poisoning," The American Journal of Emergency Medicine, vol. 29, no. 3, pp. 261-264, 2011.

[13] N. B. Hampson, C. A. Piantadosi, S. R. Thom, and L. K. Weaver, "Practice recommendations in the diagnosis, management, and prevention of carbon monoxide poisoning," American Journal of Respiratory and Critical Care Medicine, vol. 186, no. 11, pp. 1095-1101, 2012.

[14] S. R. Thom, R. L. Taber, I. I. Mendiguren, J. M. Clark, K. R. Hardy, and A. B. Fisher, "Delayed Neuropsychologic Sequelae After Carbon Monoxide Poisoning: Prevention by Treatment With Hyperbaric Oxygen," Annals of Emergency Medicine, vol. 25, no. 4, pp. 474-480, 1995.

[15] Y. Gurkan, H. Canatay, A. Toprak, E. Ural, and K. Toker, "Carbon monoxide poisoning - a cause of increased QT dispersion," Acta Anaesthesiologica Scandinavica, vol. 46, no. 2, pp. 180-183, 2002.

[16] M. Eroglu, O. Uz, Z. Isilak, M. Yalcin, A. O. Yildirim, and E. Kardesoglu, "Carbon monoxide poisoning increases TpeakTend dispersion and QTc dispersion : cardiovascular topic," Cardiovascular Journal of Africa, vol. 25, no. 3, pp. 106-109, 2014.

[17] N. B. Akilli, E. Akinci, H. Akilli et al., "A new marker for myocardial injury in carbon monoxide poisoning: $\mathrm{T}$ peak- $\mathrm{T}$ end," The American Journal of Emergency Medicine, vol. 31, no. 12, pp. 1651-1655, 2013.

[18] V. Hanci, H. Ayoğlu, S. Yurtlu et al., "Effects of acute carbon monoxide poisoning on the P-wave and QT interval dispersions," Anadolu Kardiyoloji Dergisi, vol. 11, no. 1, pp. 48-52, 2011.

[19] M. L. Dallas, Z. Yang, J. P. Boyle et al., "Carbon monoxide induces cardiac arrhythmia via induction of the late $\mathrm{Na}+$ current," American Journal of Respiratory and Critical Care Medicine, vol. 186, no. 7, pp. 648-656, 2012.

[20] K. Fagher, P. Katzman, and M. Löndahl, "Hyperbaric oxygen therapy reduces the risk of QTc interval prolongation in patients with diabetes and hard-to-heal foot ulcers," Journal of Diabetes and its Complications, vol. 29, no. 8, pp. 1198-1202, 2015.

[21] P. O'donnell, P. J. Buxton, A. Pitkin, and L. J. Jarvis, “The magnetic resonance imaging appearances of the brain in acute carbon monoxide poisoning," Clinical Radiology, vol. 55, no. 4, pp. 273-280, 2000.

[22] T. Hullin, J. Aboab, K. Desseaux, S. Chevret, D. Annane, and W. Durante, "Correlation between clinical severity and different non-invasive measurements of carbon monoxide concentration: A population study," PLoS ONE, vol. 12, no. 3, p. e0174672, 2017.

[23] H. E. Persson, G. K. Sjöberg, J. A. Haines, and J. P. De Garbino, "Poisoning severity score. Grading of acute poisoning," Journal of Toxicology - Clinical Toxicology, vol. 36, no. 3, pp. 205-213, 1998.

[24] I. M. Onvlee-Dekker, A. C. H. De Vries, and A. D. J. Ten Harkel, "Carbon monoxide poisoning mimicking long-QT induced syncope," Archives of Disease in Childhood, vol. 92, no. 3, pp. 244-245, 2007. 


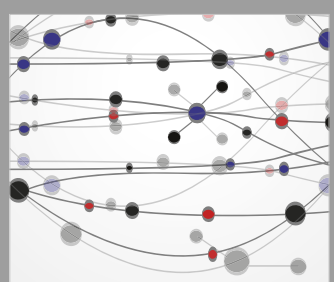

The Scientific World Journal
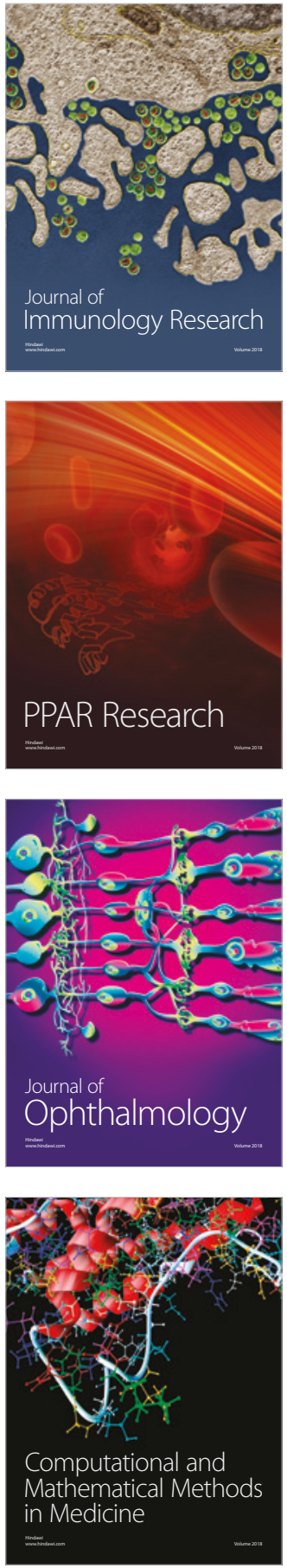

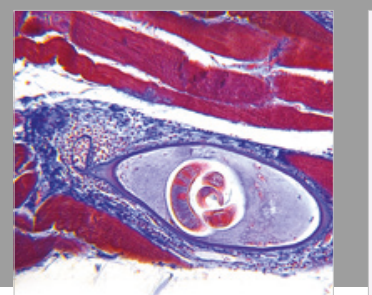

Gastroenterology Research and Practice

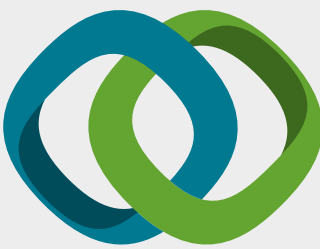

\section{Hindawi}

Submit your manuscripts at

www.hindawi.com
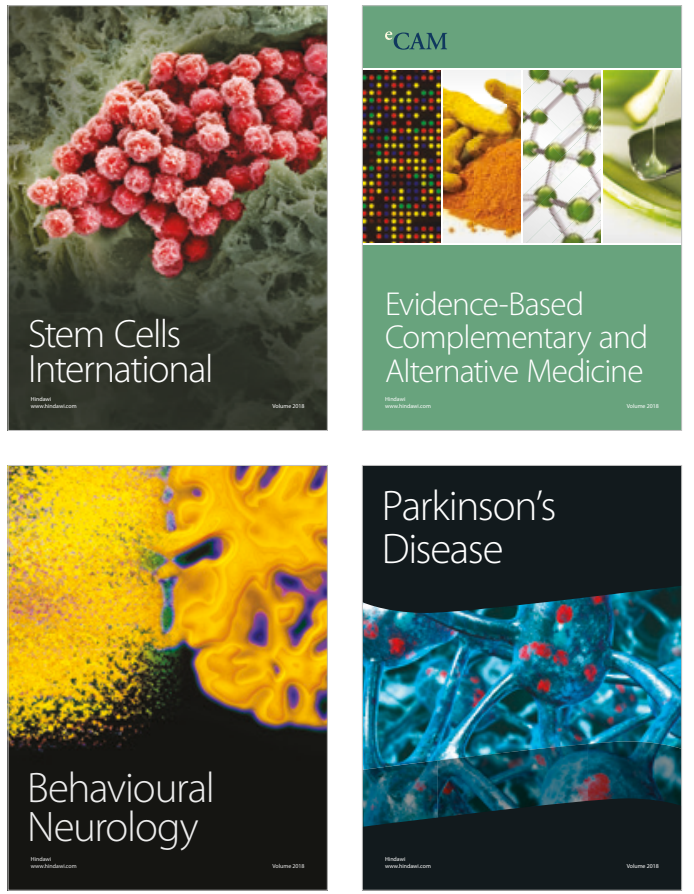

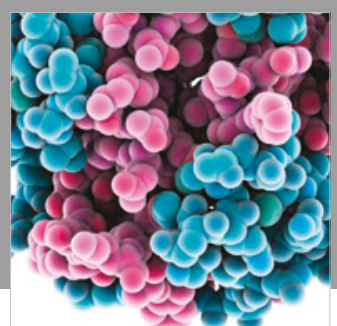

ournal of

Diabetes Research

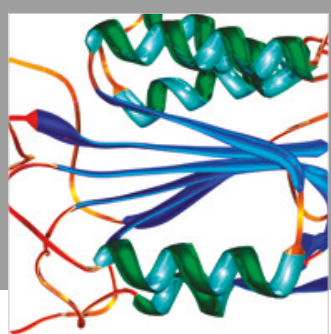

Disease Markers
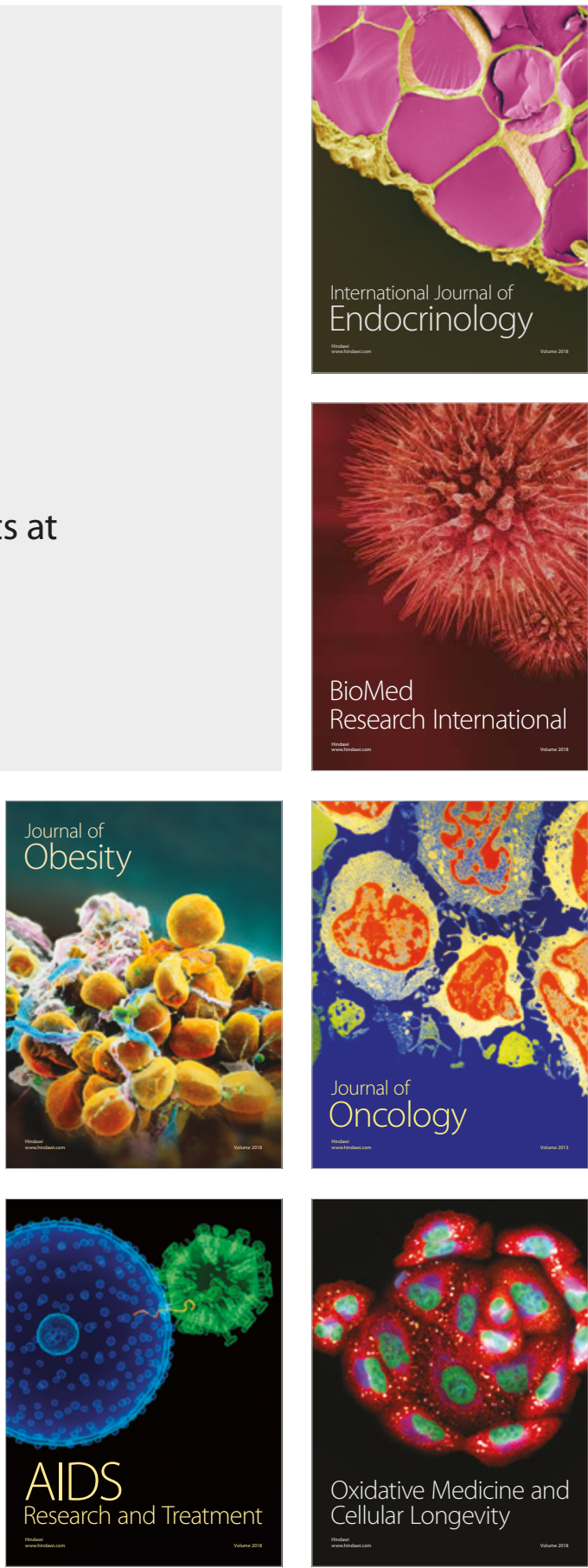